\title{
Analysis of Wavelets with Watermarking through Wavelet Transformation
}

\author{
Manupriya \\ Student \\ Amity University, India
}

\author{
Tarun Kumar \\ Assistant Professor \\ Vidya College Of Engineering, India
}

\begin{abstract}
Digital Watermarking has become essential in today's scenario because lots of digital data has become distributed all over the internet. The access to internet has become very simple and inexpensive for users in the past 15 years due to the rapid technological advancement. Therefore the possibility for the various digital attacks like forgery, damaging the digital data, stealing, copying and alteration of the digital information has also increased. In such situations, it has become significant to use a protecting mechanism that can protect the digital data and its copyright protection of the owner of digital data. Digital watermarking is a very efficient solution to this problem. Digital watermarking is a technique that embeds a watermark in the host digital data that stores the copyright information about the data. The proposed watermarking scheme is implemented in frequency domain using wavelet transform. In this paper, $\mathrm{db}$ wavelets are analyzed for the watermarking schemes. There are $45 \mathrm{db}$ wavelets, each of them is analyzed with respect to watermarking scheme and resultant $\mathrm{db}$ wavelets are given as the output which wavelets are supporting the watermarking scheme. Each of the resultant wavelet is tested against the quality parameter such as Mean Square Error, Maximum Difference, and Normalized Cross Correlation, Structural Count, and Normalized Absolute Error and Peak-Signal-To-Noise ratio.
\end{abstract}

\section{Keywords}

Watermarking, Embedding Algorithm, Extraction Algorithm, Discrete Wavelet Transform, Db wavelets

\section{INTRODUCTION}

Watermarking is a technique that is being used now-a-days for protecting the copyrights of digital assets. It is used to hide data and information with digital multimedia as it has become very important to secure the digital assets those have become spread all over the internet in a very large amount and quantity. There are various kinds of digital multimedia such as digital images, audio, digital video and various digital documents are being watermarked to enhance the security of digital assets of the respective owner. Watermarking is a very efficient technique that adds undetectable copyright information of the owner to the digital asset and thus this watermarked asset cannot be forged by any intruder.

Watermarking algorithm offers great advantages in copy control, deterrence to copying, protection of copyrights of the owner and for various authentication purposes in various applications. Watermarking technique provide an additional layer of security beyond the existing security technology. If we talk about the cryptography then a risk is there that once a digital data is decrypted by means of anything then the intruder can make use of it independently. But if the watermarking techniques are used then it is better to protect the digital data more efficiently with additional security. There is a lot of research is going on in finding the ways to make the watermark more secure and resistant to various malicious attacks [7].
In a watermarking scheme, there are two types of algorithms are used, firstly the embedding algorithm and secondly the extracting algorithm. In embedding algorithm, the host image and the watermark are accepted to be embedded. A watermark could be an image or any text. In embedding [1] the watermark is incorporated in the host image, thus this process protects the host image by embedding the watermark into it as only the owner knows what the actual watermark is, thus anyone else could not claim that watermarked data as his own. Therefore embedding algorithm is the process of locking the digital data with the watermark image or a text.

The second algorithm is the extraction algorithm which is basically used for extracting the watermark from the watermarked image. As the extraction [1] is applied to the watermarked image and the watermark image/text is extracted from the host image, it is quite natural that there is some difference in quality of the original host image and the image after the extraction process. Therefore an efficiency of a watermarking algorithm is judged on the basis of various quality parameters such as normalized correlation, peak-signal-to-noiseratio, mean square error, similarity ratio etc.

Watermarking is a very efficient approach for the copyright protection of the owner for their digital multimedia. Now there are mainly two kinds of digital watermarking technique. The first one is the visible watermarking [3] and the other one is invisible watermarking. In the visible watermarking the watermark or the logo is shown on the watermarked image and in invisible watermarking technique, the watermark or the logo is incorporated into the cover image in such a way that it does not appear clearly on the watermarked image.

Here it is very important to note that the implementation of the visible watermarking technique is easier as compared to the implementation of the invisible watermarking technique [5]. But we cannot ignore the aspect that the security provided by the invisible watermarking technique is comparatively higher than the security provided by the invisible watermarking technique.

Visible watermarking [3] techniques are used when the aim is just the presentation of the logo on the digital multimedia. But invisible watermarking provides a great security when it is important for the owner to protect its digital multimedia data from various malicious attacks and protects its digital multimedia data from various kinds of threats.

\section{LITERATURE SURVEY}

Kekre [1] proposes a novel technique for watermarking that makes use of DCT wavelet transform and it applies on various types of colour images. This algorithm selects middle frequency coefficients to embed the watermark. The aim to select the middle frequency coefficient is to enhance the security and the robustness of the image. As we know in lossy image compression high frequency coefficients can be eliminated from the watermarked image. Therefore using the middle frequency 
coefficient could enhance the robustness of the algorithm against attacks like lossy image compression.

Khalid, Deris, Kamaruddin Malik [2] proposes a watermarking technique with use of Sudoku against salt and pepper noise. This technique makes use of permutation property of Sudoku. Redundant copies of watermark are distributed to the various parts of the cover image. This technique uses the $9 * 9$ classic Sudoku puzzle. This technique uses the redundant copies of watermark image on the cover image and also the randomization is used to produce better security against the salt and pepper noise. Khalid [2] proposes an algorithm to prevent salt and pepper noise.

Khaled, Loukhaoukha [3] this technique makes use of the discrete wavelet transform and single valued decomposition along with multiobjective ant colony optimization technique. Single valued decomposition is used for decomposing the binary watermark. The singular values are then embedded in a detailed sub-band of host image. Discrete Wavelet transform is used for decomposing the host image in various frequency sub-bands.

G. Dayalin Leena [4] proposes a technique of digital watermarking that uses wavelet transforms. It mainly focuses on the use of chaotic map technique that is used to shuffle watermark image with a color logo. Mainly the frequencies of wavelet decomposition of color image are watermarked with the color logo.

Here chaotic map technique makes the embedding procedure highly secure as it is very difficult to find out the shuffling pattern imposed by chaotic map techniques for an unauthorized person. As a measure for checking the accuracy of the image, peak signal to noise ratio is used in this algorithm. Chaotic map technique adds the additional layer of security to the embedding process of this technique, as the color logo that has been used as the watermark is shuffled using chaotic map technique thus it becomes difficult for the anonymous user to detect the watermark that has been embedded into the host image. Both the images, the host image and the logo image or watermark have been taken as the color images in this technique.

Cuiwei He [5] provides a method of digital watermarking of the digital images by means of the use of, digital wavelet transform, singular value decomposition and Arnold scrambling. It proposes a method of combining these techniques and provides a new method and implements this technique in an effective way and provided a robust digital watermarking technique.

Mona M. Soliman [6] proposes watermarking techniques especially for the protection of medical images. This algorithm mainly makes use of particle swarm optimization technique in adaptive quantization index modulation. Along with this technique this algorithm also makes use of the combination of discrete wavelet transform along with single valued decomposition technique and discrete cosine transform.

Raval [8] proposes a semi-blind watermarking technique. In this technique, lower frequency component and the higher frequency component is calculated and then a circular watermark is embedded in the lower frequency and the other in the higher frequency. Their work is inspired by discrete wavelet transform technique. The base behind embedding one of them into lower frequency and the other in higher frequency is that by embedding watermarks in the low frequency component, it shows robustness against one set of attacks, while embedding in the high frequency components is robust to another set of attacks.
Murty, Bindu [10] provides a method that is a combination of discrete wavelet transform, discrete cosine transform and single valued distribution. Three watermarking techniques are proposed by the combination of discrete wavelet transform, discrete cosine transform and singular value decomposition. First watermarking technique is designed with the use of combination of discrete cosine transform and singular value decomposition, the second technique is designed with the combination of discrete wavelet transform and singular value decomposition and the third proposal of watermarking uses the combination of discrete cosine transform, discrete wavelet transform and the singular value decomposition.

Daren Huang [12] proposes a multiband wavelet transformation based watermarking technique; the empirical mode decomposition is also used in the proposed watermarking technique. In the given watermarking scheme, the watermark is incorporated in the wavelet domain in the mean-trend of middle frequencies. To provide a better robustness suitable filters are used. The dilation factor is also utilized to improve the robustness of the watermarking algorithm.

Preeti Kalra [14] provides the idea the basic function of watermarking scheme. It also suggests the different types of watermark, that is, invisible and visible, in detail. The process of watermarking along with the embedding process and the extraction process is depicted. Here it provides one more essential characteristics of a watermarking scheme apart from the other important characteristics that has been discussed above. This addition characteristic is the transparency that is the process of embedding must not decrease the quality of the watermark when it is extracted from the host image. The watermark should be clear and transparent even after the extraction process.

\section{THE PROPOSED ALGORITHM}

\subsection{Discrete Wavelet Transform}

In two dimensional discrete wavelet transform, digital data is decomposed in four frequency sub-bands. Discrete wavelet transform decompose the original cover image into four frequency sub-bands namely LL, HL, LH and HH. Here LL frequency sub-band constitutes the approximation details. The frequency sub-band LH is used to constitute the vertical details of the image, HL contains the horizontal details of the image and the HH sub-band contains the diagonal details of the image. The LL sub-band that is the approximation of the digital image could be further decomposed with the use of discrete wavelet transform to get any level of decomposition of the digital content and it will produce the further four sub-bands. Thus multiple levels of decomposition could be obtained by applying the discrete wavelet transform on the approximation part, that is, on the LL part of the digital content as desired by the application.

\subsection{Embedding Algorithm-}

There are various steps are involved in the embedding procedure. In the embedding algorithm basically the watermark information is embedded into the original host image. Embedding Architecture is shown in Figure 1.

Step 1: Convert the RGB components of the original host image into grey level components.

Step 2: Normalize the host image.

Step 3: Apply the Wavelet filters.

Step 4: Apply the wavelet transformation to decompose the host image into its frequency sub-bands LL1, LH1, HL1, and HH1.

Step 5: Apply the wavelet transformation to the LL1 sub-band to get the second level of decomposition. 
Step 6: Apply the wavelet transform to the LL2 sub-band to get the third level of decomposition.

Step 7: Convert the RGB components of watermark image into grey level components.

Step 8: Normalize the Watermark image.

Step 9: Attain three levels of decomposition of watermark using wavelet transform and get the frequency sub-bands as L_L2, L_H2, H_L2, and H_H2.

Step 10: Embed the watermark information into the host image frequency sub-bands.

Step 11: Apply the three levels of inverse wavelet transform to get the watermarked image.

\subsection{Extracting Algorithm-}

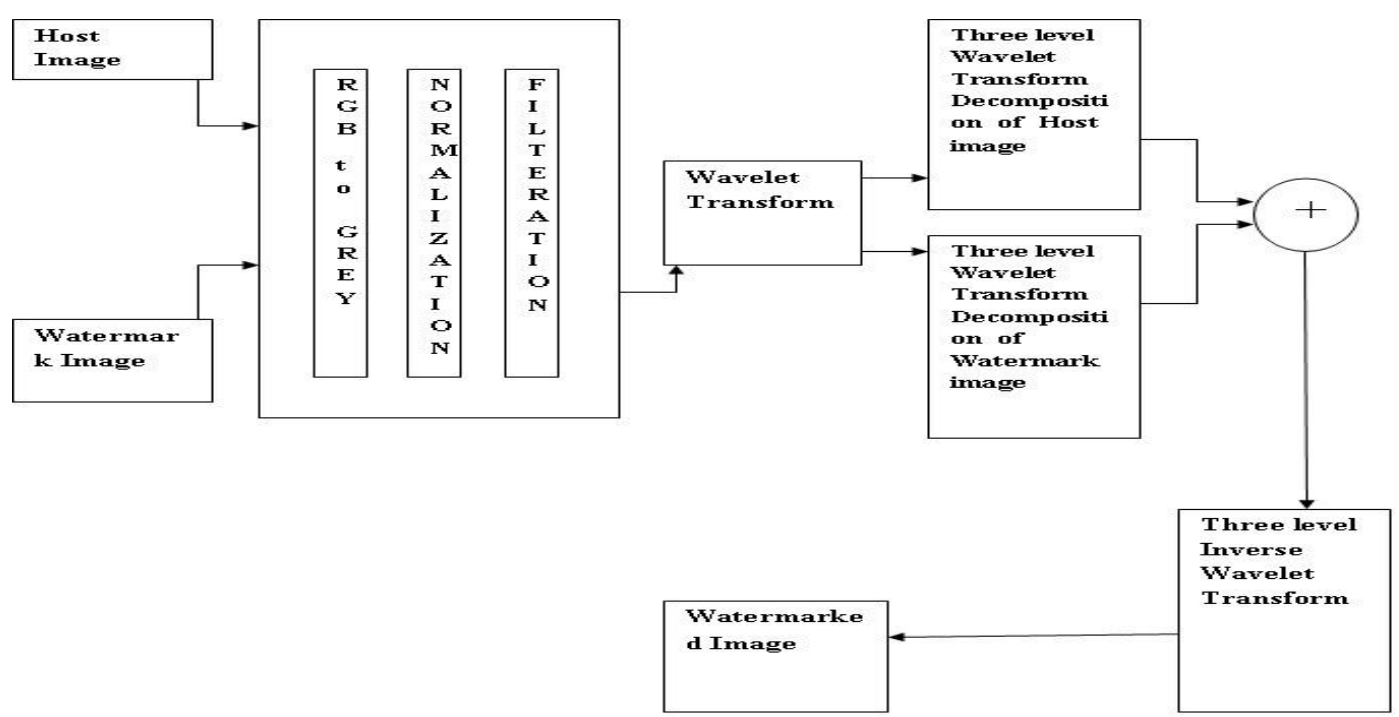

Figure 1: Embedding Architecture

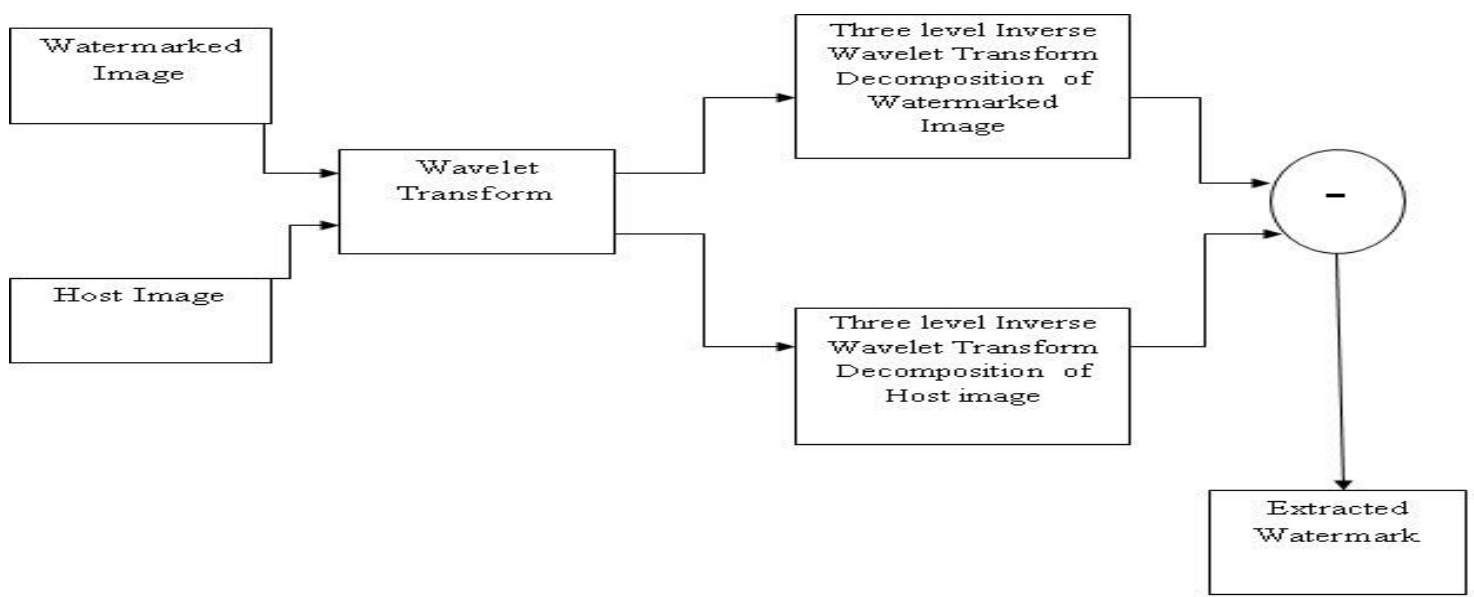

Figure 2: Extraction Architecture

3.4 Db wavelets- The db wavelets are the orthogonal wavelets those define the discrete wavelet transform and categorized by a maximal number of vanishing moments for some support. There is a scaling function given for each $\mathrm{db}$ wavelet that is used to create an orthogonal multiresolution analysis. As the Haar wavelet transform is defined, in the same manner, $\mathrm{db}$ wavelets are described. Db wavelets are defined by calculating differences and the averages by means of scalar
The extraction procedure of the watermarking technique involves extracting the watermark image that has been embedded to the cover image from the watermarked image. The extraction Architecture is shown in Figure 2. The extraction of the watermark involves several steps. These steps are explained in the extraction algorithm.

Step 1: Apply the wavelet transform to decompose watermarked image into frequency sub-bands.

Step 2: Apply the wavelet transform to the lower frequency subband of the watermarked image.

Step 3: Apply the third level of decomposition using wavelet transform to the lower frequency sub-band of the output of step

Step 4: Differentiate between the LL2 sub-band of watermarked image and the third level decomposed lower frequency sub-band of the cover image to retrieve the watermark. 
longer supports than that of haar wavelet transform. That means, $\mathrm{db}$ wavelet transform calculate the differences and averages by means of more values are used in $\mathrm{db}$ wavelet transform, but this few change, gives an exceptional improvement in its transformation capabilities. Db wavelet transform are highly useful in compression applications and eliminating the noise from the digital data such as audio and images. They are utilized in image enhancement and signal recognition also.

\section{EXPERIMENTAL RESULTS}

The images of equal size with equal number of rows and columns are taken since we are considering the square matrix. The cover image and the watermark image those are used for implementing the proposed watermarking scheme are shown as follows:
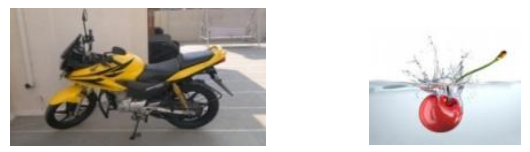

Figure 4: The Cover Image Figure 5: Watermark

\subsection{Quality Parameters}

There are following quality parameters on the basis of which deviation between the original image and the resultant image is calculated:

4.1.1 PSNR- PSNR[15] stands for Peak Signal-to-Noise Ratio. PSNR for a noise-free $m \times n$ image I and its noisy approximation $\mathrm{K}$ is given then Mean Square Error could be defined as:

$$
\begin{gathered}
\text { MSE }=\frac{1}{m n} \sum_{i=0}^{m-1} \sum_{j=0}^{n-1}[I(i, j)-K(i, j)]^{2} \\
\text { The PSNR is could be expressed as: } \\
\text { PSNR }=10 \cdot \log _{10}\left(\frac{\mathrm{MAX}^{2}}{\mathrm{MSE}}\right) \\
=20 . \log _{10}\left(\frac{\mathrm{MAX}}{\sqrt{\mathrm{MSE}}}\right) \\
=20 . \log _{10}(\mathrm{MAX})-10 . \mathrm{lc}
\end{gathered}
$$

4.1.2 Mean Square Error- The mean square error [15] could be defined as:

$$
\text { MSE }=\frac{1}{m n} \sum_{i=0}^{m-1} \sum_{j=0}^{n-1}[I(i, j)-K(i, j)]^{2}
$$

4.1.3 Normalized Cross Correlation- Normalized cross correlation [15] can be expressed as follows:

$$
\frac{\sum_{(i, j) \in W} I_{1}(i, j) \cdot I_{2}(x+i, y+j)}{\sqrt[2]{\sum_{(i, j) \in W} I_{1}^{2}}(i, j) \cdot \sum_{(i, j) \in W} I_{2}^{2}(x+i, y+j)}
$$

4.1.4 Maximum Difference- Maximum difference [15] could be expressed as follows:

$$
\begin{gathered}
\operatorname{MD}=\operatorname{Max}(\mid \text { Aij-Bij } \mid), \\
i=1,2, m \\
j=1,2, n
\end{gathered}
$$

4.1.5 Normalized Absolute Error- Normalized Absolute error [15] could be defined as follows:
The size of original image is $2048 \mathrm{X} 2048$ and the same is taken for the watermark image. The proposed watermarking technique is implemented in MATLAB.

As there are $45 \mathrm{db}$ wavelets in total, for each of the $\mathrm{db}$ wavelet, the proposed watermarking scheme is executed. After the execution of the watermarking scheme for each of the wavelet from the group of 45 wavelets, as a result $12 \mathrm{db}$ wavelets are found out that are workable for the proposed watermarking scheme. These db wavelets are db1, db5, db9, db13, db17, db21,

\begin{tabular}{|c|c|c|}
\hline Wavelet & $\begin{array}{l}\text { Watermarked } \\
\text { image }\end{array}$ & $\begin{array}{l}\text { Extracted } \\
\text { Watermark }\end{array}$ \\
\hline Db1 & & \\
\hline Db9 & & as \\
\hline Db 17 & & as \\
\hline Db25 & & os \\
\hline Db33 & & \\
\hline
\end{tabular}
$\mathrm{db} 25, \mathrm{db} 29, \mathrm{db} 33, \mathrm{db} 37, \mathrm{db} 41$, and db45.

$$
\frac{\sum_{\mathrm{i}=1}^{\mathrm{m}} \sum_{\mathrm{j}=1}^{\mathrm{n}}\left(\left|\mathrm{A}_{\mathrm{ij}}-\mathrm{B}_{\mathrm{ij}}\right|\right)}{\sum_{\mathrm{i}=1}^{\mathrm{m}} \sum_{\mathrm{j}=1}^{\mathrm{n}}\left(\mathrm{A}_{\mathrm{ij}}\right)}
$$

4.1.6 Structural Content- The Structural content [15] could be defined as follows:

$$
\frac{\sum_{\mathrm{i}=1}^{\mathrm{m}} \sum_{\mathrm{j}=1}^{\mathrm{n}}\left(\mathrm{A}_{\mathrm{ij}}{ }^{2}\right)}{\sum_{\mathrm{i}=1}^{\mathrm{m}} \sum_{\mathrm{j}=1}^{\mathrm{n}}\left(\mathrm{B}_{\mathrm{ij}}{ }^{2}\right)}
$$

The result for the proposed watermarking scheme for some of the resultant $\mathrm{db}$ wavelets could be seen in table 1 .

Table 1: Results for some $\mathrm{db}$ wavelets for the proposed watermarking technique

The values of the above quality parameters are calculated separately for each of the working $\mathrm{db}$ wavelet from $\mathrm{db} 1$ to $\mathrm{db} 45$ for the proposed watermarking scheme. The values for different quality parameters could be seen in table 2 .

Table 2: Comparison of various db wavelets on the basis of quality parameters

\begin{tabular}{|l|l|l|l|l|l|l|}
\hline $\begin{array}{l}\text { Wavel } \\
\text { ets }\end{array}$ & MSE & NCC & MD & SC & NAE & $\begin{array}{l}\text { PSN } \\
\text { R }\end{array}$ \\
\hline Db1 & 50.99 & 0.999 & 8.150 & $1.0000 \mathrm{e}+0$ & 0.999 & 2.646 \\
& 15 & 9 & 1 & 08 & 9 & 0 \\
\hline Db5 & 51.26 & 0.999 & 8.395 & $1.0000 \mathrm{e}+0$ & 0.999 & 3.185 \\
& 81 & 9 & 5 & 08 & 9 & 3 \\
\hline Db9 & 51.51 & 0.999 & 8.585 & $1.0000 \mathrm{e}+0$ & 0.999 & 3.584 \\
& 10 & 9 & 1 & 08 & 9 & 5 \\
\hline Db13 & 51.74 & 0.999 & 8.697 & $1.0000 \mathrm{e}+0$ & 0.999 & 3.799 \\
& 34 & 9 & 5 & 08 & 9 & 8 \\
\hline Db17 & 51.96 & 0.999 & 8.867 & $1.0000 \mathrm{e}+0$ & 0.999 & 4.143 \\
& 70 & 9 & 4 & 08 & 9 & 5 \\
\hline Db21 & 52.18 & 0.999 & 8.735 & $1.0000 \mathrm{e}+0$ & 0.999 & 3.801 \\
& 23 & 9 & 2 & 08 & 9 & 8 \\
\hline Db25 & 52.38 & 0.999 & 9.043 & $1.0000 \mathrm{e}+0$ & 0.999 & 4.455 \\
& 90 & 9 & 5 & 08 & 9 & 9 \\
\hline & & & & & & \\
\hline
\end{tabular}




\begin{tabular}{|l|l|l|l|l|l|l|}
\hline Db29 & 52.58 & 0.999 & 9.134 & $1.0000 \mathrm{e}+0$ & 0.999 & 4.618 \\
& 76 & 9 & 5 & 08 & 9 & 5 \\
\hline Db33 & 52.77 & 0.999 & 8.901 & $1.0000 \mathrm{e}+0$ & 0.999 & 4.064 \\
& 93 & 9 & 3 & 08 & 9 & 8 \\
\hline Db37 & 52.96 & 0.999 & 9.194 & $1.0001 \mathrm{e}+0$ & 0.999 & 4.678 \\
& 50 & 9 & 9 & 08 & 9 & 6 \\
\hline Db41 & 53.14 & 0.999 & 9.308 & $9.9885 \mathrm{e}+0$ & 0.999 & 4.890 \\
& 72 & 9 & 8 & 07 & 9 & 6 \\
\hline Db45 & 53.32 & 0.999 & 9.013 & $5.2401 \mathrm{e}+0$ & 0.999 & 4.212 \\
& 26 & 9 & 2 & 07 & 9 & 5 \\
\hline
\end{tabular}

\section{MATHEMATICAL MODEL}

We can generalize the behavior of twelve $\mathrm{db}$ wavelets those are supporting the proposed watermarking scheme. Therefore we can do the generalization of equation which can be helpful to identify which wavelet is useful for the proposed watermarking technique. Mathematical equation for this generalization could be given as:

For n wavelets,

$$
=\mathrm{db}(4 \mathrm{n}-3)
$$

For $\mathrm{n}=1,2,3, \ldots ., 12$, resultant $\mathrm{db}$ wavelets are $\mathrm{db} 1, \mathrm{db} 5, \mathrm{db} 9$, db13, db17, db21, db25, db29, db33, db37, db41 and db45.

\section{CONCLUSION}

Digital watermarking has become an essential mechanism for the protection of the digital information that has been widespread over the internet and also for the protection of the copyrights of the owner. In this paper, the analysis of various $\mathrm{db}$ wavelets has been done with respect to various quality parameters such as Mean Square Error, Normalized Cross Correlation, Maximum Difference, Structural Content, Normalized Absolute Error, and Peak Signal-to-Noise Ratio. There are $45 \mathrm{db}$ wavelets in total in which it has been found that $\mathrm{db} 1, \mathrm{db} 5, \mathrm{db} 9, \mathrm{db} 13, \mathrm{db} 17, \mathrm{db} 21$, $\mathrm{db} 25, \mathrm{db} 29, \mathrm{db} 33, \mathrm{db} 37, \mathrm{db} 41$ and $\mathrm{db} 45$ are supporting the watermarking scheme.

The values for the different quality parameters have also be calculated for these twelve db wavelets. If the Mean Square Error is the major priority of the application, then $\mathrm{db} 1$ is producing good results. If the maximum difference is having the higher priority for the application then $\mathrm{db} 1$ is producing the good results. If the structural count is a major concern for the application, then $\mathrm{db} 1, \mathrm{db} 5, \mathrm{db} 9, \mathrm{db} 13, \mathrm{db} 17, \mathrm{db} 21, \mathrm{db} 25, \mathrm{db} 29$, $\mathrm{db} 33$ may be used for providing better results. If the PSNR is the major concern then db41 is producing the better results as compared to that of other $\mathrm{db}$ wavelets.

\section{FUTURE PROSPECTS}

Digital watermarking is the area in which a lot of research is going on as it is very efficient technique for the copyright protection of the digital asset which is widespread over the internet. In this paper, a detailed analysis of $\mathrm{db}$ wavelets has been provided in an effective way. There is a huge scope of further research with the use of these $\mathrm{db}$ wavelets. The proposed watermarking scheme is analyzed for various $\mathrm{db}$ wavelets in terms of digital images. Further these wavelets could produce good results for the digital multimedia such as audio and video. More future work could be done towards the betterment of the values for the various quality parameters. The comparison of these $\mathrm{db}$ wavelets could also be given in terms of different sizes of the digital multimedia and also with respect to time.

\section{REFERENCES}

[1] H. B. Kekre, Tanuja Sarode, Shachi Natu 2014, "Robust Watermarking Scheme using Column DCT Wavelet Transform under Various Attacks", International Journal on Computer Science and Engineering (IJCSE), 2014.

[2] Shamsul Kamal Ahmad Khalid, Mustafa Mat Deris, Kamaruddin Malik Mohamad,2013"A Robust Digital Image Watermarking against Salt and Pepper using Sudoku", SDIWC ISBN: 978-0-9891305-2-3, 2013.

[3] Khaled Loukhaoukha 2013," Image Watermarking Algorithm Based on Multiobjective Ant Colony Optimization and Singular Value Decomposition in Wavelet Domain", Hindawi Publishing Corporation Journal of Optimization Volume 2013

[4] G. Dayalin Leena, S. Selva Dhayanithy 2013,” Robust Image Watermarking in Frequency Domain", International Journal of Innovation and Applied Studies ISSN 2028-9324 Vol. 2 No. 4 Apr. 2013.

[5] Cuiwei He1, Mohammad Reza Asharif1, Carlos Enrique Gutierrez1 Mahdi Khosravy, Katsumi Yamashita3 and Rui Chen4," A New Robust Image Watermarking Algorithm Based On DWT-SVD AND Arnold Scrambling", ICIC International c 2013 ISSN 1881-803X Volume 7, Number 4 April 2013.

[6] Mona M. Soliman, Aboul Ella Hassanien, Neveen I. Ghali, Hoda M. Onsi 2012," An adaptive Watermarking Approach for Medical Imaging Using Swarm Intelligent", International Journal of Smart Home Vol. 6, No. 1, January, 2012.

[7] Jonathan Blake 2011 , Shahram Latifi," Digital Watermarking Security", Defence Science Journal, Vol. 61, No. 5, September 2011, pp. 408-414,2011.

[8] Raval, M.S., Rege, P.P. 2003, "Discrete wavelet transform based multiple watermarking scheme", Conference on Convergent Technologies for Asia-Pacific Region, TENCON 2003, vol. 3, pp. 935 - 938, 15- 17 Oct. 2003.

[9] Manjit Thapa, Sandeep Kumar Sood 2011," On Secure Digital Image Watermarking Techniques", Journal of Information Security, 2011

[10] Satyanarayana Murty.P, Hima Bindu. R, Rajesh Kumar. P 2012,"A Novel Semi-Blind Reference Color Image Watermarking using DWT-DCT-SVD", International Journal of Computer Applications (0975 - 8887) Volume 53- No.15, September 2012.

[11] Rama Seshagiri Rao Channapragada, Anil Srimanth Mantha, Munaga V.N.K. Prasad," Study Of Digital Watermarking Techniques", IJCSI International Journal of Computer Science Issues, Vol. 9, Issue 6, No 1, November 2012

[12] Ning Bi, Qiyu Sun, Daren Huang, Zhihua Yang, and Jiwu Huang," Robust Image Watermarking Based on Multiband Wavelets and Empirical Mode Decomposition", IEEE TRANSACTIONS ON IMAGE PROCESSING, VOL. 16, NO. 8, AUGUST 2007.

[13] Evelyn Brannock, Michael Weeks, Robert Harrison," The Effect of Wavelet Families on Watermarking", Journal of Computers, VOL. 4, NO. 6, JUNE 2009.

[14] Dr. Ajit, Preeti Kalra, Sonia Dhull,” Digital Watermarking”, International Journal of Advanced Research in Computer 
Science and Software Engineering, Volume 3, Issue 4, April 2013.

[15] Dr.S.S.Bedi1, Mrs.Jyoti Agarwal2, Pankaj Agarwal3, "Image Fusion Techniques and Quality Assessment Parameters for Clinical Diagnosis: A Review", International Journal of Advanced Research in Computer and Communication Engineering Vol. 2, Issue 2, February 2013.
[16] I. R. Farah, I. B. Ismail, and M. B. Ahmed," A Watermarking System Using the Wavelet Technique for Satellite Images", World Academy of Science, Engineering and Technology Vol: 2 2008-05-28. 